\title{
Parkinsonian symptoms in normal pressure hydrocephalus: a population-based study
}

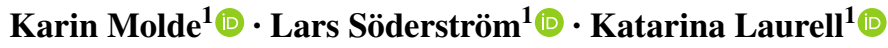

Received: 3 June 2017 / Revised: 17 August 2017 / Accepted: 18 August 2017 / Published online: 6 September 2017

(C) The Author(s) 2017. This article is an open access publication

\begin{abstract}
It may be challenging to differentiate normal pressure hydrocephalus (NPH) from neurodegenerative disorders such as Parkinson's disease. In this populationbased study, we wanted to describe the frequency of parkinsonian symptoms among individuals with and without $\mathrm{NPH}$, and whether the motor examination part of the Unified Parkinson's Disease Rating Scale (UPDRS-m) score differs between these groups. Furthermore, we wanted to find out whether there was a relationship between UPDRS-m score, NPH symptoms, and radiological signs of NPH. A sample of 168 individuals over the age of 65 with and without self-reported symptoms of NPH underwent a computerized tomography of the brain and clinical examination, including UPDRS-m to grade parkinsonian symptoms. According to diagnostic guidelines, 38 fulfilled criteria for NPH, whereas 130 had unlikely NPH. Bradykinesia was significantly more common among those with NPH (79\%) compared to those with unlikely NPH $(32 \%)(p<0.001)$. The corresponding figures for rigidity were 43 vs. $15 \%$ ( $p<0.001$ ), for postural instability 71 vs. $22 \%(p<0.001)$, and for tremor at rest 5 vs. $6 \%$ (not significant). The total UPDRS-m score was significantly higher among individuals with NPH $($ median $=12)$ than without (median $=1$ ) and correlated significantly with the degree of NPH symptoms ( $r=-0.72)$ and ventriculomegaly $(r=0.31)$. In this study, parkinsonian symptoms, except resting tremor, were frequent in individuals with NPH and correlated with the severity of NPH symptoms. Asymmetric symptoms were uncommon. We recommend a
\end{abstract}

Karin Molde

karin.molde@ regionjh.se

1 Department of Pharmacology and Clinical Neuroscience, Umeå University, Umeå, Sweden liberal use of neuroradiological imaging when investigating a patient with parkinsonian features.

Keywords Normal pressure hydrocephalus . Hydrocephalus · Parkinsonism · Parkinson's disease · UPDRS

\section{Introduction}

Parkinsonian symptomatology exists in addition to the classical triad of gait, cognitive, and urinary symptoms in normal pressure hydrocephalus (NPH) [1-4], and may complicate the diagnostic considerations [5, 6]. Radiology of the brain is essential to diagnose NPH [7], with typical findings of dilated ventricles without any macroscopic obstruction to cerebral spinal fluid (CSF) flow, often with signs of compressed cortical sulci combined with focally enlarged sulci [8]. NPH is a treatable condition; in about $80 \%$ of the patients, the symptoms improve after surgical treatment with CSF shunt [9].

The Unified Parkinson's Disease Rating Scale (UPDRS) [10] is a widely used rating scale for Parkinson's disease (PD) [11] and the motor examination part (UPDRS-m) has also been used to rate parkinsonian motor symptoms in NPH patients [12-15]. In the previous hospital-based studies, upper body bradykinesia has been described in $62 \%$ and parkinsonism in up to $71 \%$ of NPH patients $[2,12]$. Significant improvements in the total UPDRS-m score have been described after shunt surgery and after CSF removal by lumbar puncture (CSF tap test) [12-15]. Mild parkinsonian signs [16] in the four categories bradykinesia, tremor at rest, rigidity, and postural/gait changes are found in $20-40 \%$ of the older population $[17,18]$. Except from neurodegenerative diseases, factors associated with normal aging as well as 
comorbidities such as cerebrovascular disease and essential tremor may contribute to findings of isolated parkinsonian signs $[16,19]$.

As parkinsonian features are important in the differential diagnosis of neurological disorders, we wanted to describe the frequency of such symptoms and compare the UPDRS-m score between individuals from the general population with and without signs of NPH. Furthermore, we wanted to find out whether there was a relationship between UPDRS-m score, NPH symptoms, and radiological signs of NPH.

\section{Methods}

\section{Material}

This study is part of an ongoing epidemiological study on the prevalence of NPH. Out of the total population of 28,000 individuals aged 65 years or older living in Jämtland County, 1000 were randomly selected from the Swedish population register and received a questionnaire on NPH symptoms. The questionnaire is based on an on-line screening tool for NPH [20], and consists of seven yes or no questions regarding balance and gait disturbance, cognitive impairment, and urinary symptoms. Individuals who reported two symptoms (including gait or balance disturbance) or more were invited to undergo further investigations. In total, 673 returned a correct filled in questionnaire giving a response rate of $67.3 \%$, of which 168 individuals with and without symptoms of NPH underwent computerized tomography (CT) of the brain and neurological examinations. The flow chart describes the selection of the final study population (Fig. 1). Exclusion criteria were severe medical conditions sufficient to explain the symptoms, for example known brain tumor or severe multiple sclerosis diagnosed by a neurologist. Among those who returned the questionnaire and accepted further studies, one reported that he was under treatment for idiopathic PD at the Neurology Department. He had no signs of $\mathrm{NPH}$ on a previous CT scan and was excluded from further studies. Among the investigated 168 individuals, two had tested dopaminergic treatment but discontinued because of lack of effect. One of them had an ischemic lesion in basal ganglia on CT brain, whereas the other had radiological signs of NPH and received a shunt in June 2016 with a clear improvement.

To diagnose NPH, we used the guidelines by Mori et al. [7], which are suitable for population-based studies, because they can be used without CSF pressure measurement. The criteria for "possible NPH" require at least two symptoms from the clinical triad; gait disturbance, cognitive impairment, and urinary symptoms. Ventricular dilation (Evans' index $>0.3$ ) is mandatory. "Probable NPH" requires in addition to the criteria for "possible NPH", a CSF pressure of
$200 \mathrm{mmH}_{2} \mathrm{O}$, or less. However, when CSF pressure measurements are not performed, as in the present study, the diagnosis "possible NPH with neuroradiological support" can be used instead. It requires an NPH-specific radiological picture of the brain with narrowing of the sulci and subarachnoid spaces over the high convexity/midline surface [21]. Finally, the diagnosis "definitive NPH" is used when the symptoms improve after shunt surgery. To simplify, we denominated the groups as "unlikely", "possible", and "probable" NPH where the latter was equivalent to "possible NPH with neuroradiological support". The only two individuals with the diagnosis of "definite NPH", i.e., confirmed with shunt surgery were included in the "probable NPH" group.

Radiological evaluation was made with a CT scan of the brain. Previously described radiological markers were analyzed, i.e., Evans' index, callosal angle, signs of narrow medial sulci, focally enlarged sulci, dilated fissure Sylvii, and size of temporal horns [8] (Fig. 2).

To investigate the degree of parkinsonian motor symptoms, UPDRS-m [10] was used, grading the patients motor function from 0 to 4 , where 0 represents normal and 4 represents severe impairment. Tremor, rigidity, and bradykinesia in extremities are measured and scored bilaterally. A senior consultant in neurology with assistance of a trained medical student made all the examinations. Tremor at rest, bradykinesia in extremities, rigidity, and postural instability were described as present when scored 1 or more. Parkinsonism was defined according to the UK brain bank criteria, as the presence of bradykinesia together with at least one of the symptoms tremor at rest, rigidity, and postural instability [22].

We used a scale developed by Hellström et al. [23] to measure the severity of NPH symptoms. The scale is composed of four different domain scores (gait, balance, neuropsychology, and continence) which are assessed by $10 \mathrm{~m}$ walking test, ordinal ratings of gait and balance, Grooved pegboard test, Ray Auditory Verbal Learning Test (RAVLT), Stroop test, and an ordinal continence scale based on selfreports [23]. A total NPH score is calculated as the mean of the four domain scores with the gait domain counted twice. A score of 100 means the absence of symptoms, and 0 is the most severe state.

The neurologist was blinded to radiological data and, accordingly, the radiologist was blinded to clinical data.

\section{Statistical analysis}

Descriptive statistics were used to present the frequency of different parkinsonian symptoms among individuals with and without signs of NPH.

Differences in the level of parkinsonian symptoms and UPDRS score between the three groups, "unlikely", 
Fig. 1 Flow chart of sample selection. Final study population, $n=168$

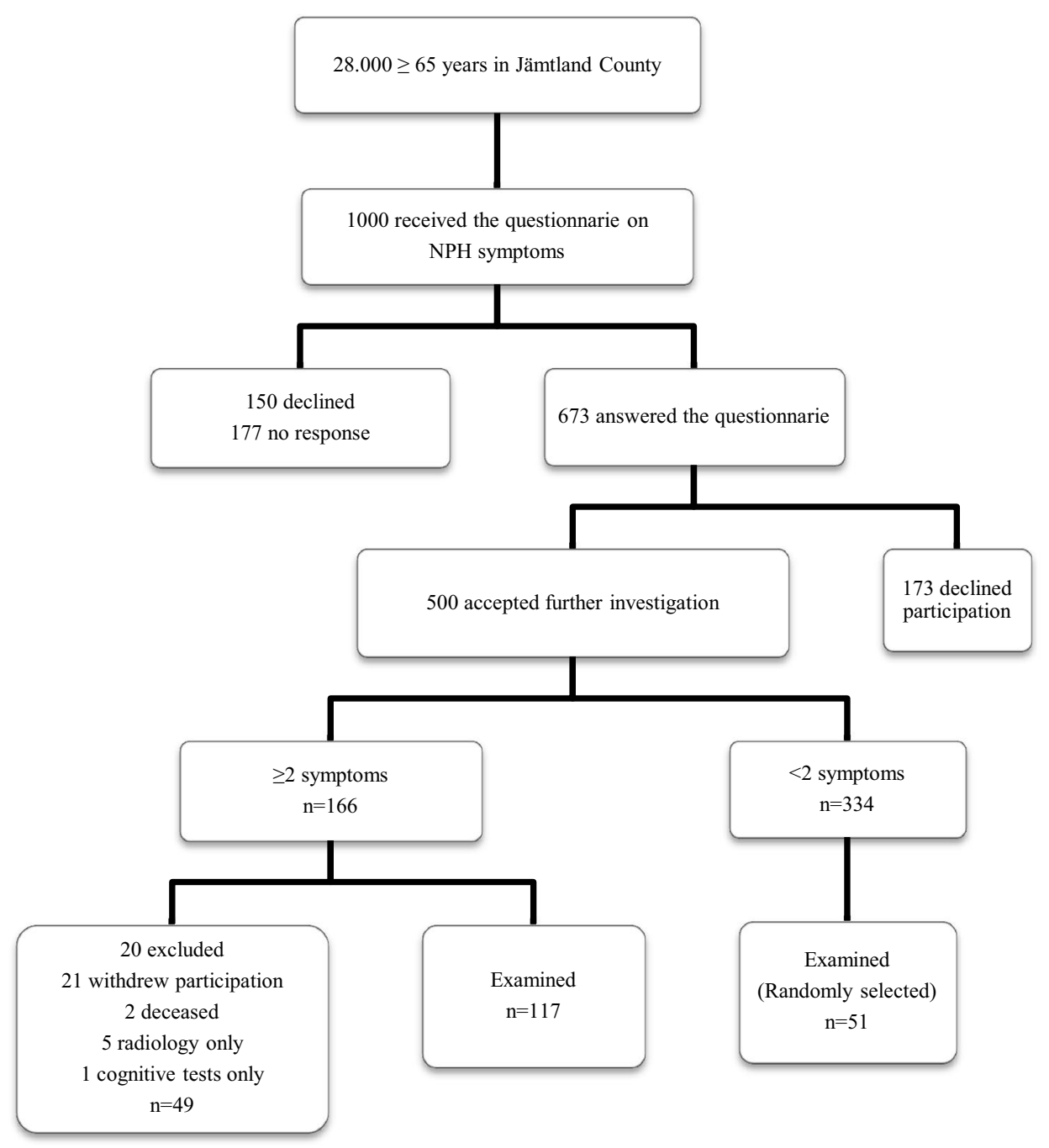

"possible", and "probable" NPH, were tested with the Fisher-Freeman-Halton exact test and Kruskal-Wallis statistical test, respectively.

Spearman correlation analyses were used to measure the association between UPDRS-m score and NPH score and the UPDRS-m score and continuous radiological variables (i.e., Evans' index, callosal angle, and size of temporal horns), respectively. The level of significance was set to $p<0.05$.

All analyses were performed using SPSS (IBM SPSS Statistics for Macintosh, Version 24.0, IBM Corp.).

\section{Ethical approval}

The Regional Ethical Review Board in Umeå approved the study (Dnr 2014/180-31) and all participants gave written, informed consent.

\section{Results}

The sample consisted of 168 individuals, 75 men and 93 women, mean 75 (66-92) years. According to diagnostic criteria, 11 individuals had "probable NPH", 27 individuals had "possible NPH", and 130 had "unlikely NPH". Table 1 shows the demography of the study population.

The frequency of specific parkinsonian symptoms according to NPH diagnosis is shown in Table 2. Among those with NPH (i.e., "possible NPH" and "probable NPH”), bradykinesia was found in $79 \%$, of which in upper extremities in $68 \%$. The corresponding figures for tremor at rest were $5 \%$, rigidity $43 \%$, and postural instability $71 \%$, respectively. Parkinsonism was found in $71 \%$ of those with NPH, and in $20 \%$ of those with unlikely NPH.

Except for facial expression and tremor, the UPDRS-m scores differed significantly between the groups of "unlikely", "possible", and "probable" NPH (Table 3). As expected, 

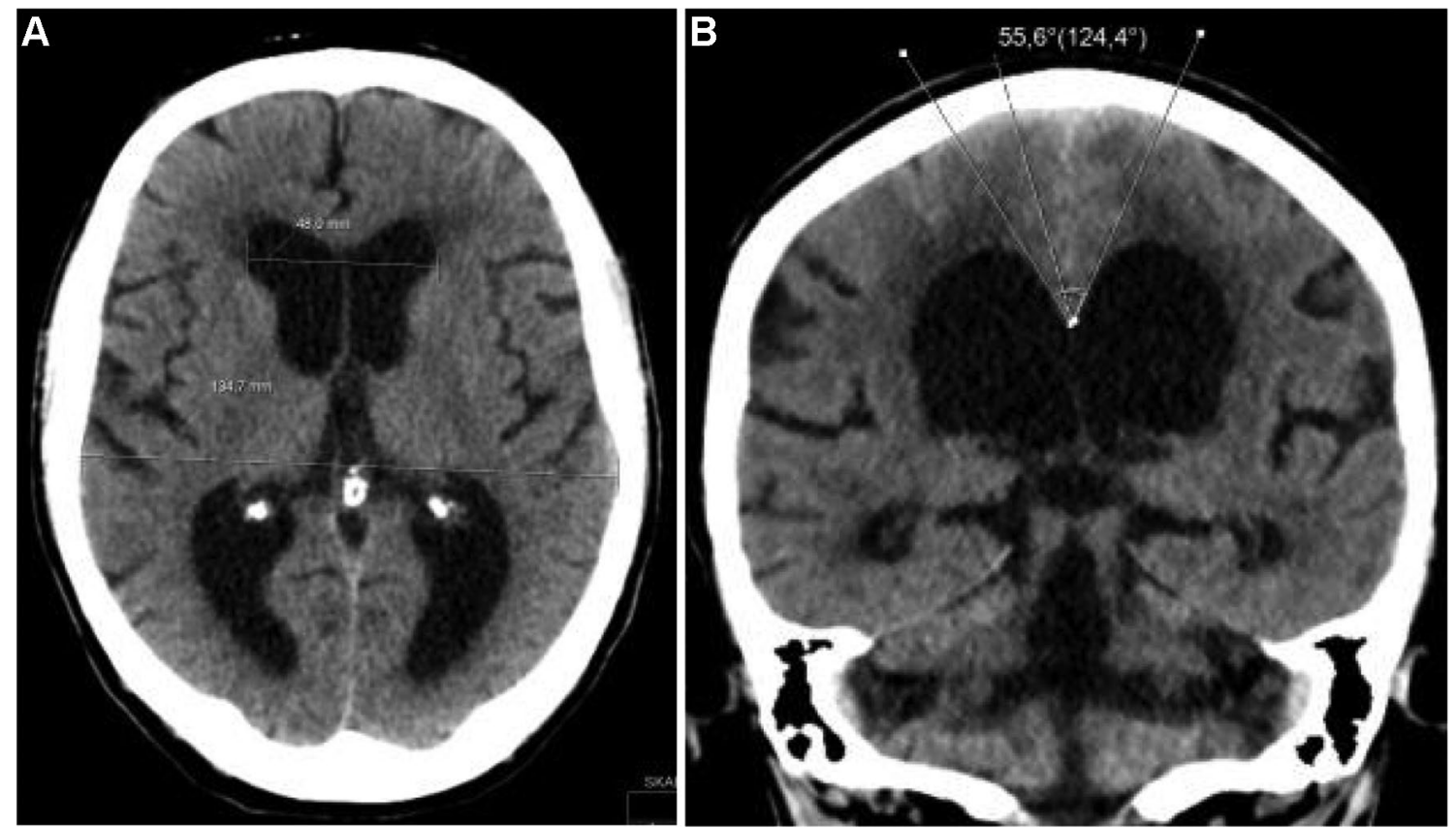

Fig. 2 Radiological markers of NPH shown on CT scan a Evans' index (ratio of maximum width of the frontal horns to the maximum inner skull diameter) $>0.3$; $\mathbf{b}$ callosal angle $<60^{\circ}$, narrow medial sulci, dilated fissure Sylvii, and dilated temporal horns

Table 1 Demography of the study population

\begin{tabular}{llll}
\hline & $\begin{array}{l}\text { Unlikely NPH } \\
(n=130)\end{array}$ & $\begin{array}{l}\text { Possible NPH } \\
(n=27)\end{array}$ & $\begin{array}{l}\text { Prob- } \\
\text { able NPH } \\
(n=11)\end{array}$ \\
\hline$n$ male (\%) & $51(39)$ & $16(59)$ & $8(73)$ \\
Mean age (SD) & $74(5.87)$ & $79(7.65)$ & $80(7.29)$ \\
\hline
\end{tabular}

NPH normal pressure hydrocephalus

the difference was most marked between individuals with "unlikely" and "probable" NPH, and further pronounced for the total UPDRS-m score, where those without NPH had a median score of $1(0-23)$ and those with NPH (i.e., "possible" and "probable" NPH) had a median score of 12 (0-35).

The score of NPH symptoms [23] correlated significantly with score on the UPDRS-m $(r=-0.72, p<0.001)$ (Fig. 3). Furthermore, the radiological markers Evans' index and size of temporal horns correlated significantly with the UPDRS$\mathrm{m}$ score $(r=0.31$ and $r=0.39, p<0.001)$, whereas the radiological marker callosal angle $(r=-0.11)$ did not.

\section{Discussion}

In this population-based study, bradykinesia and rigidity were more than twice, and postural instability more than three times as frequent in individuals with NPH than in those without NPH. In contrast, tremor at rest was unusual and similar between the two groups. The total UPDRS-m score was significantly higher among individuals with NPH and correlated to the severity of NPH symptoms. Our findings confirm the results from the previous studies on hospitalbased material that parkinsonism can be a part of the clinical syndrome of NPH [2, 3, 12-15].

Of the 38 individuals with NPH, $71 \%$ showed parkinsonism according to the UK Brain Bank Criteria [22]. This equals the frequency of parkinsonism (71\%) reported in a study of 17 shunt-responsive (definitive) NPH patients [12], where the definition of parkinsonism was the presence of at least two symptoms out of bradykinesia, tremor at rest, rigidity, or postural instability. With the same definition, the frequency of parkinsonism would only be slightly higher (74\%) in our material. In our NPH sample, upper body bradykinesia was slightly higher (68 vs. $62 \%$ ) and rigidity three times as prevalent (43 vs. $14 \%$ ) compared to a hospital-based study of $65 \mathrm{NPH}$ patients who improved after CSF removal and were considered for surgery [2]. This might be explained by that the cut-off level for bradykinesia was lower in our study (UPDRS-m score $=1$ ) than in their study (UPDRS-m score $=2$ ), and that we, in contrast to them, included paratonia in the assessment of rigidity. However, one also has to consider a selection bias in clinical material in that patients with parkinsonian features might be less often considered for shunt surgery.

Parkinsonian signs were also present in the group with "unlikely NPH" where we found bradykinesia in one-third of the individuals evaluated, rigidity in one out of seven, 
Table 2 Frequency of parkinsonian symptoms according to diagnosis

\begin{tabular}{llll}
\hline & $\begin{array}{l}\text { Unlikely NPH }(n=130) \\
n(\%)\end{array}$ & $\begin{array}{l}\text { Possible NPH }(n=27) \\
n(\%)\end{array}$ & $\begin{array}{l}\text { Probable NPH }(n=11) \\
n(\%)\end{array}$ \\
\hline $\begin{array}{l}\text { Bradykinesia in extremities } \\
(n=167)\end{array}$ & $41(32)$ & $20(74)$ & $10(91)$ \\
Unilateral/bilateral & $16(12) / 25(20)$ & $7(26) / 13(48)$ & $0(0) / 10(91)$ \\
Only arm/hand & $28(22)$ & $10(37)$ & $4(36)$ \\
Only leg & $3(2)$ & $3(11)$ & $1(9)$ \\
Tremor at rest & $8(6)$ & $1(4)$ & $1(9)$ \\
Unilateral/bilateral & $3(2) / 1(1)$ & $0(0) / 1(4)$ & $1(9) / 0(0)$ \\
Only arm & $4(3)$ & $0(0)$ & $1(9)$ \\
Only leg & $0(0)$ & $0(0)$ & $0(0)$ \\
Head/face tremor & $7(5)$ & $0(0)$ & $0(0)$ \\
Rigidity $(n=162)$ & $19(15)$ & $6(25)$ & $9(82)$ \\
Unilateral/bilateral & $5(4) / 14(11)$ & $2(8) / 4(17)$ & $4(36) / 5(46)$ \\
Only arm & $8(6)$ & $2(8)$ & $1(9)$ \\
Only leg & $4(3)$ & $0(0)$ & $0(0)$ \\
Postural instability & $29(22)$ & $18(67)$ & $9(82)$ \\
\hline
\end{tabular}

$N P H$ normal pressure hydrocephalus

Table 3 Differences in UPDRS-m score between "unlikely", "possible”, and "probable” NPH

\begin{tabular}{|c|c|c|c|c|c|c|c|}
\hline & \multicolumn{2}{|c|}{ Unlikely NPH $(n=130)$} & \multicolumn{2}{|c|}{ Possible NPH $(n=27)$} & \multicolumn{2}{|c|}{ Probable NPH $(n=11)$} & \multirow[t]{2}{*}{$p$ value } \\
\hline & Mean \pm SD & Median (range) & Mean \pm SD & Median (range) & Mean \pm SD & Median (range) & \\
\hline Speech & $0.02 \pm 0.20$ & $0(0-2)$ & $0.00 \pm 0.00$ & $0(0-0)$ & $0.27 \pm 0.65$ & $0(0-2)$ & 0.002 \\
\hline Facial expression & $0.05 \pm 0.23$ & $0(0-1)$ & $0.11 \pm 0.32$ & $0(0-1)$ & $0.18 \pm 0.41$ & $0(0-1)$ & 0.197 \\
\hline Tremor at rest ${ }^{\mathrm{a}}$ & $0.09 \pm 0.40$ & $0(0-3)$ & $0.15 \pm 0.77$ & $0(0-4)$ & $0.09 \pm 0.30$ & $0(0-1)$ & 0.829 \\
\hline Action or postural tremor ${ }^{b}$ & $0.39 \pm 1.11$ & $0(0-6)$ & $0.85 \pm 1.59$ & $0(0-6)$ & $0.18 \pm 0.40$ & $0(0-1)$ & 0.094 \\
\hline Rigidity $(n=162)^{\mathrm{c}}$ & $0.42 \pm 1.29$ & $0(0-8)$ & $0.83 \pm 1.74$ & $0(0-6)$ & $2.82 \pm 2.32$ & $2(0-7)$ & $<0.001$ \\
\hline Finger taps ${ }^{\mathrm{b}}$ & $0.32 \pm 0.77$ & $0(0-4)$ & $0.93 \pm 1.14$ & $0(0-4)$ & $1.09 \pm 1.04$ & $1(0-3)$ & $<0.001$ \\
\hline Hand movements ${ }^{\mathrm{b}}$ & $0.33 \pm 0.77$ & $0(0-4)$ & $0.81 \pm 1.04$ & $0(0-3)$ & $1.18 \pm 0.87$ & $1(0-2)$ & $<0.001$ \\
\hline Rapid alternating movements ${ }^{\mathrm{b}}$ & $0.37 \pm 0.86$ & $0(0-4)$ & $1.11 \pm 1.43$ & $0(0-4)$ & $1.82 \pm 1.08$ & $2(0-3)$ & $<0.001$ \\
\hline Leg agility ${ }^{\mathrm{b}}$ & $0.18 \pm 0.57$ & $0(0-3)$ & $0.67 \pm 1.04$ & $0(0-4)$ & $1.27 \pm 1.42$ & $1(0-4)$ & $<0.001$ \\
\hline Rising from chair $(n=167)$ & $0.26 \pm 0.63$ & $0(0-3)$ & $0.67 \pm 0.88$ & $0(0-3)$ & $1.00 \pm 0.89$ & $1(0-2)$ & $<0.001$ \\
\hline Posture $(n=167)$ & $0.27 \pm 0.51$ & $0(0-3)$ & $0.52 \pm 0.64$ & $0(0-2)$ & $1.09 \pm 0.94$ & $1(0-3)$ & $<0.001$ \\
\hline Postural stability & $0.29 \pm 0.60$ & $0(0-3)$ & $1.00 \pm 1.00$ & $1(0-4)$ & $1.36 \pm 1.12$ & $1(0-4)$ & $<0.001$ \\
\hline Gait & $0.43 \pm 0.81$ & $0(0-3)$ & $1.04 \pm 0.76$ & $1(0-3)$ & $1.82 \pm 0.87$ & $2(1-3)$ & $<0.001$ \\
\hline Body bradykinesia $(n=167)$ & $0.22 \pm 0.50$ & $0(0-2)$ & $0.93 \pm 0.73$ & $1(0-2)$ & $1.64 \pm 0.81$ & $2(0-3)$ & $<0.001$ \\
\hline Total score, $\max =108(n=162)$ & $3.55 \pm 5.41$ & $1(0-23)$ & $9.00 \pm 6.87$ & $8(0-25)$ & $15.82 \pm 8.35$ & $15(3-35)$ & $<0.001$ \\
\hline
\end{tabular}

UPDRS- $m$ Unified Parkinson's Disease Rating Scale-motor examination part, $N P H$ normal pressure hydrocephalus

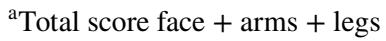

${ }^{\mathrm{b}}$ Items measured bilaterally show score right + left

${ }^{\mathrm{c}}$ Total score neck + arms + legs

and postural instability in one out of five. These results are similar to those found in the previous population-based studies $[17,18]$.

The pathophysiology of NPH is not fully understood. Theories involve a high resistance to CSF outflow contributing to ventricular enlargement, mechanical pressure of the brain parenchyma, disturbance of cerebral blood flow $(\mathrm{CBF})$, and increased water content in periventricular areas $[24,25]$. In NPH patients, significantly reduced CBF in the thalamus, the head of caudate nucleus and putamen has been shown with positron emission tomography (PET) [26]. A study with fluorodopa PET in a patient with obstructive 


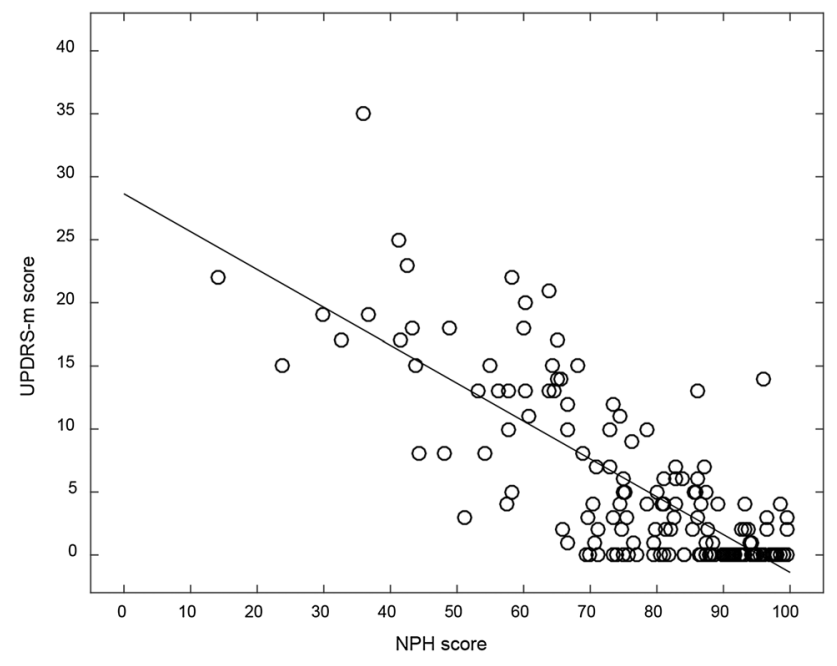

Fig. 3 Scatterplot, illustrating the association between the NPH score $(x$-axis) and UPDRS-m score ( $y$-axis) among the 168 study participants, $r=-0.72, p<0.001$

hydrocephalus who developed parkinsonism due to shunt dysfunction revealed reduced uptake in the caudate and putamen [27]. Further indications of a disturbance in the nigrostriatal pathway were provided in a recent study of 30 patients with NPH and parkinsonism where striatal dopaminergic deficit on dopamine transporter (DaT) scan was found in almost half of the patients [14]. Whether such disturbances are due to global or regionally reduced CBF [25, $26,28-30]$ or other mechanisms is not fully clear. A reduction in postsynaptic $\mathrm{D} 2$ receptors binding in the putamen of NPH patients has been demonstrated [31], and there are theories of changes in additional dopaminergic pathways as well that also contribute to parkinsonism in NPH [2, 12, 32].

The reversibility of parkinsonian signs that has been reported after shunt surgery or CSF removal supports that these symptoms are caused by hydrocephalus and not just a result of comorbidity [12-15]. A recent hospital-based study with $55 \mathrm{NPH}$ patients who underwent a CSF tap test revealed a significant improvement of bradykinesia in upper and lower bodies, whereas tremor marginally improved and rigidity did not improve [15]. In a recent publication, oral dopaminergic therapy added a positive effect to shunt surgery in the improvement of the UPDRS-m score in patients with NPH and parkinsonism, indicating that the disturbance in the dopaminergic system might not be totally reversible [14].

The findings in the present study highlight some of the diagnostic challenges when meeting a patient with parkinsonian features. However, there are some clinical hallmarks that can be useful. In contrast to PD [22], tremor at rest seems uncommon among individuals with NPH [2], and according to our results, asymmetric symptomatology is less common as well. In PD, non-motor symptoms such as olfactory dysfunction and rapid eye movement (REM) sleep behavior disorder are common and can precede the motor symptoms [33], and such symptoms would be valuable to study also in NPH patients to see if their presence could help in differentiating the disorders from each other [34]. Neuropsychiatric symptoms as depression and anxiety are frequent in both conditions [35]. When comparing the gait disturbance in PD and NPH, Stolze et al. [32] found reduced velocity and stride length, freezing phenomenon, and reduced cadence in both groups; in addition, NPH patients had a broad-based gait, outwardly rotated feet, and a diminished step height. Nevertheless, we agree with Bugalho et al. [36] that it might be difficult to differ PD from NPH only by gait function. Bradykinesia of the hand is showed to share the same features in NPH patients as in patients with PD [37], and even experienced neurologists might mistake these disorders $[5,6]$. In addition, the disorders may co-occur. These circumstances illustrate the importance of a liberal use of radiological investigation when the PD symptoms are atypical or do not respond to dopaminergic treatment.

Although it may complicate the diagnostic procedure, parkinsonian symptomatology in NPH should not exclude the patient from shunt surgery, as the symptoms diminish postoperatively [12-14].

The strength of this study is the relatively large, unselected sample with individuals from the general population. Only three individuals had been under investigation for NPH: one had received a shunt a few years prior to the study, one received a shunt afterwards (June 2016), and one declined operation. The shunt operated patients improved postoperatively; they were both wheelchair bound before the operation and regained walking ability.

The study has some limitations, as well. We did not exclude individuals with common comorbidities such as arthrosis and vascular disease, to minimize the risk of excluding those who suffered from NPH as well. Although this might have influenced the UPDRS-m score, we believe that it increases the generalizability of the results. This is supported by the fact that the group without NPH did not show more parkinsonian symptoms than what has been previously reported in the general population of elderly [17, 18]. We used CT scans as the neuroradiological evaluation instead of MRI which is suggested in the diagnostic guidelines [7], but this should be of minor importance as most radiological signs of NPH are seen on a CT scan of the brain as well. Likewise, we did not assess the lumbar opening pressure which, according to guidelines, should not exceed $200 \mathrm{mmH}_{2} \mathrm{O}$ in "probable NPH" [7]. However, according to clinical experience, a pressure slightly above this level is not uncommon among NPH patients, and to rule out a noncommunicating hydrocephalus, with clearly increased CSF pressure, neuroradiological imaging should be used instead. 
Finally, we do not yet know how many of those with NPH in this study will be offered and respond to shunt surgery, i.e., fulfill the diagnosis of "definitive NPH".

\section{Conclusion}

In this study, parkinsonian motor symptoms, except resting tremor, were frequent among individuals with NPH and correlated with the severity of NPH symptoms. Asymmetric distribution was rare. We recommend a liberal use of neuroradiological imaging when investigating a patient with parkinsonian features, in particular when the symptoms do not response to dopaminergic treatment.

Acknowledgements The authors want to thank the participants and their relatives in this study, the employees of the Neurology and Radiology departments at the hospital of Östersund for practical help, and the Unit of Research, Education and Development, Region Jämtland Härjedalen, for the financial support.

\section{Compliance with ethical standards}

Ethical standards The Regional Ethical Review Board in Umeå approved the study (Dnr 2014/180-31); the study has been performed in accordance with the ethical standards laid down in the 1964 Declaration of Helsinki and its later amendments.

Conflicts of interest On behalf of all authors, the corresponding author states that there is no conflict of interest.

Sources of funding Employed by the hospital of Östersund, Region Jämtland Härjedalen, Sweden (KM, KL). Employed by the Unit of Research, Education and Development, Region Jämtland Härjedalen, Sweden (LS). Employed by Umeå University, Sweden (KL). Grants from the Unit of Research, Education and Development, Region Jämtland Härjedalen, Sweden (KL). The authors report no other disclosures.

Informed consent All participants gave written, informed consent.

Open Access This article is distributed under the terms of the Creative Commons Attribution 4.0 International License (http:// creativecommons.org/licenses/by/4.0/), which permits unrestricted use, distribution, and reproduction in any medium, provided you give appropriate credit to the original author(s) and the source, provide a link to the Creative Commons license, and indicate if changes were made.

\section{References}

1. Curran T, Lang AE (1994) Parkinsonian syndromes associated with hydrocephalus: case reports, a review of the literature, and pathophysiological hypotheses. Mov Disord 9:508-520. doi: $10.1002 / \mathrm{mds} .870090503$

2. Krauss JK, Regel JP, Droste DW et al (1997) Movement disorders in adult hydrocephalus. Mov Disord 12:53-60. doi:10.1002/ mds. 870120110
3. Mandir AS, Hilfiker J, Thomas G et al (2007) Extrapyramidal signs in normal pressure hydrocephalus: an objective assessment. Cerebrospinal Fluid Res 4:7. doi:10.1186/1743-8454-4-7

4. Blomsterwall E, Bilting M, Stephensen H, Wikkelsö C (1995) Gait abnormality is not the only motor disturbance in normal pressure hydrocephalus. Scand J Rehabil Med 27:205-209

5. Jellinek EH (2008) Not Parkinson's disease: neurologists' mistakes with a diversion into adult hydrocephalus. Pract Neurol 8:322-324. doi:10.1136/jnnp.2008.152595

6. Conn HO, Lobo FM (2008) What do physicians know about normal pressure hydrocephalus and when did they know it? A survey of 284 physicians. Yale J Biol Med 81:19-29

7. Mori E, Ishikawa M, Kato T et al (2012) Guidelines for management of idiopathic normal pressure hydrocephalus: second edition. Neurol Med Chir (Tokyo) 52:775-809. doi:10.2176/ nmc. 52.775

8. Virhammar J, Laurell K, Cesarini KG, Larsson E-M (2014) Preoperative prognostic value of MRI findings in 108 patients with idiopathic normal pressure hydrocephalus. AJNR Am J Neuroradiol 58:1-8. doi:10.3174/ajnr.A4046

9. Klinge P, Hellström P, Tans J, Wikkels $\varnothing$ C (2012) One-year outcome in the European multicentre study on iNPH. Acta Neurol Scand 126:145-153. doi:10.1111/j.1600-0404.2012.01676.x

10. Fahn S, Elton RL; UPDRS Program Members (1987) Unified Parkinson's disease rating scale. In: Fahn S, Marsden CD, Goldstein M, Calne DB (eds) Recent Dev. Park. Dis., vol 2. Maximilian Healthcare Information, Florham Park, pp 153-163, 293-304

11. Ramaker C, Marinus J, Stiggelbout AM, van Hilten BJ (2002) Systematic evaluation of rating scales for impairment and disability in Parkinson's disease. Mov Disord 17:867-876. doi:10.1002/ mds. 10248

12. Akiguchi I, Ishii M, Watanabe Y et al (2008) Shunt-responsive parkinsonism and reversible white matter lesions in patients with idiopathic NPH. J Neurol 255:1392-1399. doi:10.1007/ s00415-008-0928-1

13. Ishii M, Kawamata T, Akiguchi I et al (2010) Parkinsonian symptomatology may correlate with CT findings before and after shunting in idiopathic normal pressure hydrocephalus. Parkinsons Dis. doi: $10.4061 / 2010 / 201089$

14. Broggi M, Redaelli V, Tringali G et al (2016) Normal pressure hydrocephalus and parkinsonism: preliminary data on neurosurgical and neurological treatment. World Neurosurg 90:348-356. doi:10.1016/j.wneu.2016.03.004

15. Kang K, Jeon JS, Kim T et al (2016) Asymmetric and upper body parkinsonism in patients with idiopathic normal-pressure hydrocephalus. J Clin Neurol 12:452-459. doi:10.3988/ jcn.2016.12.4.452

16. Louis ED, Bennett DA (2007) Mild Parkinsonian signs: an overview of an emerging concept. Mov Disord 22:1681-1688. doi: $10.1002 / \mathrm{mds} .21433$

17. Louis ED, Luchsinger JA, Tang MX, Mayeux R (2003) Parkinsonian signs in older people: prevalence and associations with smoking and coffee. Neurology 61:24-28. doi:10.1212/01. WNL.0000072330.07328.D6

18. Uemura Y, Wada-Isoe K, Nakashita S, Nakashima K (2011) Mild parkinsonian signs in a community-dwelling elderly population sample in Japan. J Neurol Sci 304:61-66. doi:10.1016/j. jns.2011.02.013

19. Keezer MR, Wolfson C, Postuma RB (2016) Age, gender, comorbidity, and the MDS-UPDRS: results from a population-based study. Neuroepidemiology 46:222-227. doi:10.1159/000444021

20. Factora R, Luciano M (2008) When to consider normal pressure hydrocephalus in the patient with gait disturbance. Geriatrics 63:32-37

21. Hashimoto M, Ishikawa M, Mori E et al (2010) Diagnosis of idiopathic normal pressure hydrocephalus is supported by MRI-based 
scheme: a prospective cohort study. Cerebrospinal Fluid Res 7:18. doi:10.1186/1743-8454-7-18

22. Lees AJ, Hardy J, Revesz T (2009) Parkinson's disease. Lancet 373:2055-2066. doi:10.1016/S0140-6736(09)60492-X

23. Hellström P, Klinge P, Tans J, Wikkelsø C (2012) A new scale for assessment of severity and outcome in iNPH. Acta Neurol Scand 126:229-237. doi:10.1111/j.1600-0404.2012.01677.x

24. Malm J, Eklund A (2006) Idiopathic normal pressure hydrocephalus. Pract Neurol 6:14-27. doi:10.1136/jnnp.2006.088351

25. Owler BK, Pickard JD (2001) Normal pressure hydrocephalus and cerebral blood flow: a review. Acta Neurol Scand 104:325-342. doi:10.1034/j.1600-0404.2001.00092.x

26. Owler BK, Momjian S, Czosnyka Z et al (2004) Normal pressure hydrocephalus and cerebral blood flow: a PET study of baseline values. J Cereb Blood Flow Metab 24:17-23. doi:10.1097/01. WCB.0000093326.88757.49

27. Racette BA, Esper GJ, Antenor J et al (2004) Pathophysiology of parkinsonism due to hydrocephalus. J Neurol Neurosurg Psychiatry 75:1617-1619. doi:10.1136/jnnp.2003.028449

28. Momjian S, Owler BK, Czosnyka Z et al (2004) Pattern of white matter regional cerebral blood flow and autoregulation in normal pressure hydrocephalus. Brain 127:965-972. doi:10.1093/brain/ awh131

29. Ziegelitz D, Starck G, Kristiansen D et al (2014) Cerebral perfusion measured by dynamic susceptibility contrast MRI is reduced in patients with idiopathic normal pressure hydrocephalus. J Magn Reson Imaging 39:1533-1542. doi:10.1002/jmri.24292

30. Virhammar J, Laurell K, Ahlgren A et al (2014) Idiopathic normal pressure hydrocephalus: cerebral perfusion measured with pCASL before and repeatedly after CSF removal. J Cereb Blood Flow Metab 34:1771-1778. doi:10.1038/jcbfm.2014.138

31. Ouchi Y, Nakayama T, Kanno T et al (2007) In vivo presynaptic and postsynaptic striatal dopamine functions in idiopathic normal pressure hydrocephalus. J Cereb Blood Flow Metab 27:803-810. doi:10.1038/sj.jcbfm.9600389

32. Stolze H, Kuhtz-Buschbeck JP, Drücke H et al (2001) Comparative analysis of the gait disorder of normal pressure hydrocephalus and Parkinson's disease. J Neurol Neurosurg Psychiatry 70:289-297

33. Poewe W (2008) Non-motor symptoms in Parkinson' s disease. Eur J Neurol 15(Suppl. 1):14-20

34. Passler JS, Doty RL, Dolske MC et al (2017) Olfactory ability in normal pressure hydrocephalus as compared to Alzheimer s disease and healthy controls. J Neurol Sci 372:217-219. doi:10.1016/j.jns.2016.11.049

35. Kito Y, Kazui H, Kubo Y et al (2009) Neuropsychiatric symptoms in patients with idiopathic normal pressure hydrocephalus. Behav Neurol 21:165-174. doi:10.3233/BEN-2009-0233

36. Bugalho P, Alves L, Miguel R (2013) Gait dysfunction in Parkinson's disease and normal pressure hydrocephalus: a comparative study. J Neural Transm 120:1201-1207. doi:10.1007/ s00702-013-0975-3

37. Nowak DA, Topka HR (2006) Broadening a classic clinical triad: the hypokinetic motor disorder of normal pressure hydrocephalus also affects the hand. Exp Neurol 198:81-87. doi:10.1016/j. expneurol.2005.11.003 